\title{
Reporte de altos requerimientos de insulina en pacientes críticos pediátricos con COVID-19. Experiencia con monitoreo remoto continuo de glucosa
}

\author{
Report of high insulin requirements in pediatric critically ill patients with \\ COVID-19. Experience with continuous remote glucose monitoring
}

\author{
Andrea Gabriela Krochik², Mariana Prieto², Carolina Martínez Mateu², Consuelo Barcala², \\ Rosario Gallagher ${ }^{3}$, Silvia Filippini ${ }^{3}$, Delfina Arambarri' ${ }^{4}$, Leandro Mendoza ${ }^{5}$, Nicolás Rosales ${ }^{6}$, \\ Hernán De Battista ${ }^{6}$, Ricardo Sánchez Peña ${ }^{7}$, Fabricio Garelli ${ }^{6}$
}

\section{RESUMEN}

Los pacientes en estado crítico con COVID-19 sufren hiperglucemias sostenidas de difícil manejo. A esto se suma el desafío de minimizar la exposición al contagio.

En el presente artículo analizamos la evolución metabólica de dos pacientes pediátricos con COVID-19 admitidos en unidad de cuidados intensivos (UCI) para pacientes COVID-19 del Hospital "Prof. Dr. Juan P. Garrahan" de la Ciudad Autónoma de Buenos Aires, Argentina, que requirieron tratamiento con insulina endovenosa y cuya glucemia fue monitoreada de manera remota con la plataforma InsuMate ${ }^{\circledR}$ desarrollada en la Universidad Nacional de La Plata. Los pacientes requirieron tasas de infusión de insulina en dosis marcadamente mayores que las de otros pacientes críticos que impresionaron relacionadas con los valores de marcadores de inflamación. La infusión pudo ajustarse con cuatro monitoreos diarios de glucosa y las métricas obtenidas con el monitor de glucosa. El uso del sistema de monitoreo remoto continuo de glucosa permitió disminuir la frecuencia de monitoreo glucémico durante el tratamiento.

Palabras clave: COVID-19; hiperglucemia; paciente crítico pediátrico; monitoreo remoto de glucosa.

Revista de la Sociedad Argentina de Diabetes 2021; Vol. 55 (70-74)

\section{ABSTRACT}

Critically ill patients with COVID-19 suffer from sustained hyperglycemia that is difficult to manage. Added to this is the challenge of minimizing exposure to contagion.

In this article we analyze the metabolic evolution of two pediatric patients with COVID-19 admitted to the intensive care unit (ICU) for COVID-19 patients at the Hospital "Prof. Dr. Juan P. Garrahan "from the Autonomous City of Buenos Aires, Argentina, who required treatment with intravenous insulin and whose blood glucose was remotely monitored with the InsuMate ${ }^{\circledR}$ platform developed at the National University of La Plata.

The patients required insulin infusion rates in doses markedly higher than those of other critically ill patients, who were impressively related to the values of inflammation markers. The infusion could be adjusted with four daily glucose monitors and the metrics obtained with the glucose monitor. The use of the continuous remote glucose monitoring system made it possible to decrease the frequency of glycemic monitoring during treatment.

Key words: COVID-19; hyperglycemia; pediatric critical patient; remote glucose monitoring.

Revista de la Sociedad Argentina de Diabetes 2021; Vol. 55 (70-74)
1 Médica Pediatra, especialista en Nutrición Pediátrica, Jefa de Clínica del Servicio de Nutrición, Hospital "Prof. Dr. Juan P. Garrahan", Ciudad Autónoma de Buenos Aires, Argentina

2 Médica Pediatra, especialista en Nutrición Pediátrica, Hospital "Prof. Dr. Juan P. Garrahan", Ciudad Autónoma de Buenos Aires, Argentina

3 Médica Pediatra, especialista en Terapia Intensiva Pediátrica, Hospital "Prof. Dr. Juan P. Garrahan", Ciudad Autónoma de Buenos Aires, Argentina

4 Ingeniera en Computación, Grupo de Control Aplicado, Instituto de Investigaciones en Electrónica, Control y Procesamiento de Señales (LEICI), Universidad Nacional de La Plata, Consejo Nacional de Investigaciones Científicas y Técnicas (CONICET), Provincia de Buenos Aires, Argentina

5 Estudiante de Ingeniería, Grupo de Control Aplicado, Instituto de Investigaciones en Electrónica, Control y Procesamiento de Señales (LEICI), Universidad Nacional de La Plata, Provincia de Buenos Aires, Argentina
6 Doctor en Ingeniería, Grupo de Control Aplicado, Instituto de Investigaciones en Electrónica, Control y Procesamiento de Señales (LEICI), Universidad Nacional de La Plata, Consejo Nacional de Investigaciones Científicas y Técnicas (CONICET), Provincia de Buenos Aires, Argentina

7 Doctor en Ingeniería, Centro de Sistemas y Control, Instituto Tecnológico de Buenos Aires, Consejo Nacional de Investigaciones Científicas y Técnicas (CONICET), Ciudad Autónoma de Buenos Aires, Argentina. CONICET

Contacto de la autora: Andrea Gabriela Krochik

E-mail: gabkro66@hotmail.com

Correspondencia: Combate de los Pozos 1881 (C1245AAM),

Ciudad Autónoma de Buenos Aires, Argentina

Fecha de trabajo recibido: 26/01/21

Fecha de trabajo aceptado: 07/03/21

Conflictos de interés: los autores declaran que no existe conflicto de interés 


\section{INTRODUCCIÓN}

El 11 de marzo de 2020 la COVID-19 fue declarada pandemia por la Organización Mundial de la Salud (OMS)'1. En Argentina, el primer caso se confirmó en marzo, y hasta la fecha (enero de 2020), se reportaron 1.874 .801 casos confirmados y 47.034 fallecidos.

Aunque los niños parecen tener menos probabilidades de desarrollar una enfermedad grave, se recomienda un seguimiento estricto en aquellos con comorbilidades ${ }^{2}$.

Los pacientes en estado crítico secundario a COVID-19 sufren hiperglucemia y aumento de la variabilidad glucémica ${ }^{3}$ atribuibles al uso de corticoides e inótropicos, la inflamación y la hipoxia con disminución de secreción insulínica y daño pancreático ${ }^{4,5}$, por lo cual se prolonga el tiempo de internación y aumenta la mortalidad ${ }^{6,7}$.

El tratamiento de la hiperglucemia requiere frecuentemente infusión endovenosa de insulina con monitoreos horarios de glucemia. En el caso de los pacientes críticos con COVID-19 se suma el desafío de minimizar la exposición al contagio. Recientemente se ha propuesto disminuir la frecuencia de mediciones de glucemia en estos pacientes cada 4 a 6 horas $(h)^{8,9,10}$, sin embargo esto podría no ser adecuado ni seguro.

Aunque los sistemas de monitoreo continuo de glucosa en tiempo real fueron desarrollados para el control ambulatorio, la Food and Drug Administration (FDA) autorizó en, marzo de 2020, su uso para el control remoto de la glucemia en pacientes con COVID-19'11.

\section{OBJETIVOS}

Nuestro objetivo es presentar la evolución metabólica de dos pacientes pediátricos con COVID-19 que requirieron tratamiento de hiperglucemia en unidad de cuidados intensivos (UCI), y describir la utilidad del sistema de monitoreo remoto continuo de glucosa utilizado.

\section{MATERIALES Y MÉTODOS}

Se analizaron, en forma retrospectiva, los datos de dos pacientes con COVID-19, internados en la UCl del Hospital "Prof. Dr. Juan P. Garrahan" de la Ciudad Autónoma de Buenos Aires (Argentina) durante julio y agosto de 2020, que requirieron tratamiento con insulina endovenosa y en los que se utilizó, previa firma de consentimiento familiar, un sistema de monitoreo online remoto de glu- cosa, sumado a los controles de glucemia capilar para el ajuste de la tasa de infusión de insulina.

El monitoreo online remoto se realizó mediante la plataforma InsuMate ${ }^{12}$ (https://www.insumate. com.ar// en combinación con monitores continuos de glucosa (MCG) Dexcom G6. La plataforma multicentro y multi-paciente InsuMate fue originalmente diseñada por el equipo de investigación para uso en un proyecto de páncreas artificial ${ }^{13}$ y adaptada para monitoreo en $\mathrm{UCl}$ en el contexto de la pandemia. La información recabada por cada MCG fluye hacia un teléfono celular a través de Bluetooth con una frecuencia de 5 minutos, y desde él hacia un servidor y a los dispositivos de los médicos en tiempo real vía WiFi o 4G. Una aplicación web permite a los médicos e ingenieros monitorear de manera remota la evolución de la glucemia de hasta 40 pacientes simultáneamente $24 / 7$, mientras que las métricas calculadas por la plataforma se actualizan diariamente.

\section{CASOS}

\section{Caso 1. C. J.}

Niño de 12 años de edad, con antecedente de bajo peso al nacer, patología crónica asociada y múltiples internaciones previas con síndrome de distrés respiratorio. Ingresó con dificultad respiratoria y fiebre, y se constató PCR SARS-CoV-2 (polymerase chain reaction severe acute respiratory syndrome coronavirus 2) positiva. Presentó síndrome de distrés respiratorio del adulto y neumonía por Klebsiella Pneumoniae multirresistente, con requerimiento de asistencia ventilatoria mecánica, inotrópicos y antibióticoterapia. La radiografía mostró infiltrado algodonoso bilateral. Por hiperglucemia sostenida mayor a 400 mg\%, comenzó tratamiento con infusión de insulina endovenosa, controles glucémicos cada $4 \mathrm{~h}$ y monitoreo remoto continuo de glucosa. Los antecedentes personales, datos clínicos y de laboratorio, y las métricas se muestran en Tabla 1. Las glucemias descendieron a un rango entre de 80 y 250 mg\%, y, paulatinamente, disminuyó el requerimiento insulínico a medida que mejoraron los factores inflamatorios plasmáticos lo que permitió suspender la insulinización luego de cuatro días. El dosaje de péptido $C$ fue de 10,86 ng/ml para una glucemia de 235 mg/dl (Figura 1A).

\section{Caso 2. Z. L.}

Paciente de 14 años de edad con trasplante renal de dos años de evolución, por enfermedad renal crónica de etiología desconocida, en tratamiento inmunosupresor, con obesidad y diabetes mellitus 
(DM) de un año de evolución, con anticuerpos IAA (anticuerpos anti autoinsulina), GADA (anticuerpo antidecarboxilasa del ácido glutámico), IA2 (anticuerpos anti tirosina fosfatasa 2) y ZNT8 (anticuerpos anti transportador ZNT8) negativos, en tratamiento basal/ bolo con insulina subcutánea. La HbA1c previa fue de $8,3 \%(67,4 \mathrm{mmol} / \mathrm{mol})$. Ingresó con fiebre, dolor abdominal, diarrea, deshidratación y alteración del sensorio, y se constató PCR SARS- CoV- 2 positiva y radiografía de tórax con infiltrado intersticial bilateral. Con diagnóstico de síndrome inflamatorio multisistémico, se suspendió azatioprina y recibió plasma de convaleciente. Requirió infusión de insulina endovenosa, controles de glucemia cada $4 \mathrm{~h}$ y monitoreo remoto continuo de glucosa. Los antecedentes personales, datos clínicos y de laboratorio, y las métricas se muestran en Tabla 1. La insulina endovenosa pudo suspenderse al séptimo día, en coincidencia con la mejoría clínica y la disminución de las concentraciones de factores inflamatorios plasmáticos (Figura 1B). Su péptido C fue de $2,98 \mathrm{ng} / \mathrm{ml}$ para una glucemia de $132 \mathrm{mg} / \mathrm{dl}$ y la $\mathrm{HbA} 1 \mathrm{C}$ de $10,07 \%(86,5 \mathrm{mmol} /$ mol). Durante los 20 días posteriores requirió dosis de insulina $80 \%$ mayores que sus dosis habituales.

\begin{tabular}{|c|c|c|}
\hline & Caso 1 & Caso 2 \\
\hline Sexo & Masculino & Femenino \\
\hline Edad & 12 años & 14 años \\
\hline Peso & $40 \mathrm{~kg}$ & $49 \mathrm{~kg}$ \\
\hline Diagnósticos previos & $\begin{array}{l}\text { Encefalopatía crónica epiléptica, escoliosis severa, desorden } \\
\text { deglutorio, asma }\end{array}$ & $\begin{array}{l}\text { Trasplante renal debido a enfermedad renal crónica sin } \\
\text { diagnóstico }\end{array}$ \\
\hline Historia previa de DM & No & Sí \\
\hline Tratamiento previo de DM & - & $\begin{array}{l}\text { Basal: glargina } 20 \mathrm{U} / \text { día } \\
\text { Bolus: glulisina } 24 \mathrm{U} \text { día }\end{array}$ \\
\hline Tiempo de evolución previo de DM & - & 12 meses \\
\hline $\begin{array}{l}\text { Anticuerpos para DM1 } \\
\text { (IAA, GADA, IA2, ZNT8) }\end{array}$ & - & Negativos \\
\hline Medicación previa & $\begin{array}{l}\text { Levetiracetam } 17 \mathrm{mg} / \mathrm{kg} \\
\text { Clobazam } 0,2 \mathrm{mg} / \mathrm{kg} \\
\text { Sultiamo } 3,7 \mathrm{mg} / \mathrm{kg} \\
\text { Montelukast }\end{array}$ & $\begin{array}{l}\text { Meprednisona } 4 \text { mg/día } \\
\text { Azatioprina } 50 \text { mg/día } \\
\text { Tacrolimus } 10 \text { mg/día }\end{array}$ \\
\hline Fecha de PCR para COVID-19 & $17 / 07 / 2020$ & $07 / 08 / 2020$ \\
\hline Laboratorio de ingreso & $\begin{array}{l}\text { Hemograma con } 10.710 \text { leucocitos } 51 \% \text { neutrófilos } \\
\text { segmentados, } 21 \% \text { linfocitos, } 15 \% \text { monocitos, } 8 \% \text { eosinófilos, } \\
\text { y } 523.000 \text { plaquetas } \\
\text { NT-ProBnp } 269 \mathrm{pg} / \mathrm{ml} \text {, troponina } 17 \mathrm{ng} / \mathrm{L}, \mathrm{CPK} 572 \mathrm{UI} / \mathrm{L} \text {, } \\
\text { quick } 70 \% \mathrm{KPTT} 48 \text { segundos, fibrinógeno } 392 \mathrm{mg} / \mathrm{dl} \text {, } \\
\text { PCR } 76 \mathrm{mg} / \mathrm{l} \text {, procalcitonina } 2,26 \mathrm{ng} / \mathrm{ml} \text {, LDH } 771 \mathrm{UI} / \mathrm{L}\end{array}$ & $\begin{array}{l}\text { Hemograma con } 1.870 \text { leucocitos, } 77 \% \text { neutrófilos segmentados, } \\
9 \% \text { linfocitos, } 14 \% \text { monocitos, y } 0 \% \text { eosinófilos, hemoglobina } \\
10,6 \mathrm{~g} / \mathrm{dl} \text {, plaquetas } 81,000 \mathrm{~mm} 3 \text {, fibrinógeno } 747 \mathrm{mg} / \mathrm{dl} \text {, ferritina } \\
837 \mathrm{ug} / \text {, CPK } 33 \mathrm{U} / \mathrm{l} \text {, creatinina 3,14 mg/dl, urea } 145 \mathrm{mg} / \mathrm{dl} \text {, } \\
\text { NT-ProBnp } 4.273 \mathrm{pg} / \mathrm{ml}, \text { PCR } 206,43 \mathrm{mg} / \text {, tiempo de } \\
\text { protrombina time } 117 \% \text {, KPTT } 33 \text { segundos y glucosa } 618 \mathrm{mg} / \mathrm{dl}\end{array}$ \\
\hline Fecha de admission en $\mathrm{UCl}$ & $7 / 17 / 2020$ & $8 / 12 / 2020$ \\
\hline Diagnóstico & Síndrome de distrés respiratorio del adulto & Síndrome de respuesta inflamatoria sistémica \\
\hline Días de ventilación mecánica & 42 & - \\
\hline Coinfección & Klebsiella pneumoniae, MRSA y levaduras en cultivo respiratorio & No \\
\hline $\begin{array}{l}\text { Días de administración de } \\
\text { insulina endovenosa }\end{array}$ & 4 & 6 \\
\hline $\begin{array}{l}\text { Dosis media requerida de } \\
\text { insulina/kg/día (IU) }\end{array}$ & 1,37 UI/kg/día & 1,34 UI/kg/día \\
\hline $\begin{array}{l}\text { Media de glucemia cuatro días } \\
\text { previos a monitoreo continuo }\end{array}$ & $271,59 \pm 16,4 \mathrm{mg} / \mathrm{dl}$ & $466,56 \pm 21,6 \mathrm{mg} / \mathrm{dl}$ \\
\hline $\begin{array}{l}\text { Media de glucemia con } \\
\text { monitoreo continuo }\end{array}$ & $183,5 \pm 39,3 \mathrm{mg} / \mathrm{dl}$ & $267,1 \pm 75,3 \mathrm{mg} / \mathrm{dl}$ \\
\hline $\begin{array}{l}\text { Coeficiente de variación } \\
\sin / \text { con monitoreo continuo }\end{array}$ & $60,3 \% / 21,4 \%$ & $46,2 \% / 28,2 \%$ \\
\hline $\begin{array}{l}\text { Media de controles diarios de glucemia } \\
\text { capilar sin /con monitoreo continuo }\end{array}$ & $6 / 4,1$ & $9,2 / 4$ \\
\hline $\begin{array}{l}\text { Con monitoreo continuo: } \\
\text { Tiempo de glucemia entre } 140 \\
\text { y } 180 \mathrm{mg} / \mathrm{dl} \\
\text { Tiempo de glucemia entre } 140 \\
\text { y } 250 \mathrm{mg} / \mathrm{dl} \\
\text { Tiempo de glucemia menor a } 70 \mathrm{mg} / \mathrm{dl}\end{array}$ & $\begin{array}{l}32,5 \% \\
83,9 \% \\
0 \%\end{array}$ & $\begin{array}{l}9,3 \% \\
42,7 \% \\
0 \%\end{array}$ \\
\hline
\end{tabular}

DM: diabetes mellitus; IAA: anticuerpos anti autoinsulina; GADA: anticuerpos antidecarboxilasa del ácido glutámico; IA2: anticuerpos anti tirosina fosfatasa 2; ZNT8: anticuerpos anti transportador ZNT8; UCl: unidad de cuidados intensivos; MRSA: stafilococus aureus meticilino resistente.

Tabla 1: Datos generales. 


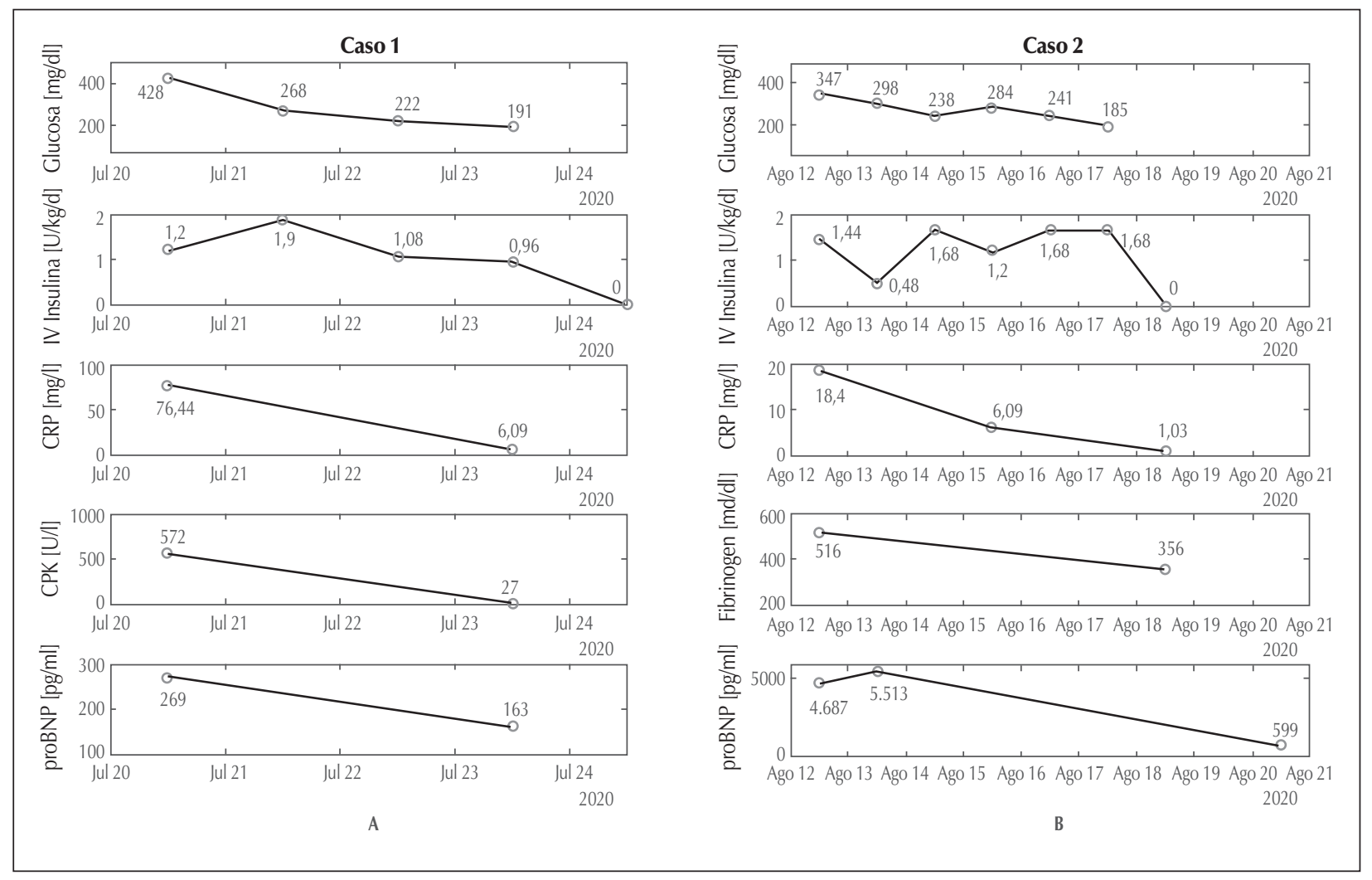

Figura 1: Curvas de promedios de glucemia, dosis de infusion de insulina y marcadores inflamatorios en pacientes 1 y 2.

\section{DISCUSIÓN Y CONCLUSIONES}

Se presentan dos pacientes pediátricos internados en $\mathrm{UCl}$, con hiperglucemia secundaria a COVID-19. Uno sin DM previa y otro con DM secundaria a trasplante renal. De acuerdo a nuestro conocimiento, éste es el primer reporte de pacientes pediátricos en cuidados intensivos por COVID-19 con hiperglucemia del paciente crítico y monitoreo de glucosa continuo a distancia.

Nuestro grupo utiliza un protocolo de insulinización en pacientes críticos para lograr el rango objetivo glucémico de 140 a 180 mg/dl, con infusiones endovenosas de insulina máximas de $0,2 \mathrm{Ul} / \mathrm{kg}$ de peso corporal/día. En coincidencia, Agus et al. ${ }^{6}$, en su estudio de pacientes pediátricos en UCI sin COVID-19, informaron un máximo de $0,14 \mathrm{UI} / \mathrm{kg}$ de peso corporal/día de insulina endovenosa para lograr objetivos glucémicos similares. Los pacientes en nuestro reporte requirieron 1,37 Ul/kg de peso corporal/día en el caso del paciente 1 y 1,34 UI/kg de peso corporal/día para la paciente 2, esta última con persistencia de la elevación de los requerimientos al pasar al tratamiento subcutáneo habitual.
En adultos con COVID-19 se describen similares patrones de hiperglucemia y requerimientos insulínicos ${ }^{14}$. No queda claro aún si se debe exclusivamente a la insulinorresistencia causada por la inflamación sistémica y la criticidad, o si algún tipo de injuria de la célula beta podría contribuir a la misma. Dado que el SARS-CoV-2 utiliza los receptores ACE2 como ingreso celular y éstos se expresan en páncreas, podría tratarse de un daño directo con alteración en la secreción insulínica ${ }^{5}$. No parece ser el caso en estos pacientes, que presentaron secreción de péptido $\mathrm{C}$ normal en el primer caso o esperable en el segundo.

La mejoría del rango glucémico al comenzar la infusión de insulina se asociaría a la posibilidad de realizar ajustes inmediatos ante cambios agudos de insulinorresistencia secundarios a la inflamación ${ }^{15}$ y a las intervenciones médicas. En el caso de los pacientes descriptos, ambos recibieron corticosteroides durante toda la internación y uno recibió inotrópicos, sin embargo, la necesidad de insulina endovenosa impresionó relacionarse principalmente con los niveles de marcadores de inflamación.

En cuanto al sistema de monitoreo de gluce- 
mia continuo, dado que aún no existen estudios que validen su seguridad y eficacia en pacientes críticos, se utilizó como suplemento y no como reemplazo del control de la glucemia capilar; aún así, permitió un monitoreo estrecho de la glucemia sin aumentar la exposición del equipo de salud. La utilización de las métricas obtenidas con el sistema de monitoreo a distancia permitió controlar y tratar la hiperglucemia, disminuir su variabilidad y prevenir episodios de hipoglucemia.

Queda aún por evaluar, en forma sistemática,

\section{BIBLIOGRAFÍA}

1. Coronavirus Resourcer Center. Johns Hopkins University and Medicine. Disponible en: https://coronavirus.jhu.edu/map.html. Último acceso: 09/09/20.

2. US Department of Health and Human Services/Centers for Disease Control and Prevention. Coronavirus Disease 2019 in Children. United States, February 12 April 2, 2020. Morbidity and mortality Weekly Report April 10, 2020; Vol. 69, Nº 14.

3. Wang A, Zhao W, Xu Z, Gu J.Timely blood glucose management for the outbreak of 2019 novel coronavirus disease (COVID-19) is urgently needed. Diabetes Research and Clinical Practice 2020; 162:108118.

4. Zhi L, et al. From the insight of glucose metabolism disorder: oxygen therapy and blood glucose monitoring are crucial for quarantined COVID-19 patients. Ecotoxicology and Environmental Safety 2020; 197:110614.

5. Wang $F$, Wang $H$, Fan J, et al. Pancreatic injury patterns in patients with COVID-19 pneumonia. Gastroenterology 2020; 159(1):367-370.

6. Agus MS, Wypij D, Hirshberg EL, et al. Tight glycemic control in critically III children. N Engl J Med 2017; 376(8)729-741.

7. Zhou J, Tan J. Diabetes patients with COVID-19 need better blood glucose management in Wuhan, China. Metabolism Clinical Experimental 2020; 107:154216.

8. Gianchandani R, Esfandiari NH, Ang L, lyengar J, Knotts S, Choksi P, Pop-Busui R. Managing hyperglycemia in the COVID-19 inflammatory storm. Diabetes 2020 Oct; 69(10):2048-2053.

9. Ceriello A, Standl E, Catrinoiu D, et al. Issues for the management of people with diabetes and COVID-19 in ICU. Cardiovasc Diabetol 2020; 19:114. la sensibilidad y exactitud de estos equipos en las condiciones clínicas de los pacientes críticos. Existe evidencia de interferencia en la exactitud de las mediciones glucémicas en pacientes que reciben numerosas drogas ${ }^{16}$.

Una de las principales limitantes consideradas inicialmente para su uso fue la posibilidad de que la introducción de una nueva tecnología interfiriera en el trabajo del equipo de salud, sin embargo, y contrariamente, se reportó un alto nivel de aceptación y utilidad del mismo.

10. Korytkowski M, Antinori-Lent K, Drincic A, et al. A pragmatic approach to inpatient diabetes managemet during the COVID-19 pandemic. J Clin Endocrinol Metab 2020; 105(9):1-12.

11. Coronavirus (COVID-19) Update: FDA allows expanded use of devices to monitor patients' vital signs remotely. Disponible en:https:/www.fda.gov/news-events/press-announcements/ coronavirus-covid-19-update-fda-allows-expanded-use-devicesmonitor-patients-vital-signs-remotely.

12. Arambarri D, Mendoza L, Rosales N, Garelli F. InsuMate: implementation and testing of Open Source Platform for Artificial Pancreas System. ATTD20-0670. Proceedings of 13th International Conference on Advanced Technology \& Treatments for Diabetes, Madrid, Spain, Feb. 2020.

13. Sánchez-Peña R, Colmegna P, Garelli F, De Battista H, et. al. Artificial pancreas: clinical study in Latin America without premeal insulin boluses. Journal of Diabetes Science andTechnology 2018; 12(5):914-925.

14. Wu L, Girgis CM, Cheung NW. COVID-19 and diabetes: insulin requirements parallel illness severity in critically unwell patients. Clin Endocrinol (Oxf) 2020 Oct; 93(4):390-393

15. Van der Crabben SN, Blümer RM, Stegenga ME, et al. Early endotoxemia increases peripheral and hepatic insulin sensitivity. J Clin Endocrinol Metab 2009; 94(2):463-68.

16. Wallia A, Umpierrez GE, Rushakoff RJ, Klonoff DC, Rubin DJ, Hill Golden S, Cook CB, Thompson B. Consensus statement on inpatient use of continuous glucose monitoring. J Diabetes Sci Technol 2017; 11:1036-1044. 